\title{
Beliefs and Information Seeking in Patients With Cancer in Southwest China: Survey Study
}

Juan Xie ${ }^{1}$, BS; Shi Xie ${ }^{2}$, BA; Ying Cheng ${ }^{1}$, PhD; Zhe $\mathrm{He}^{3}$, PhD

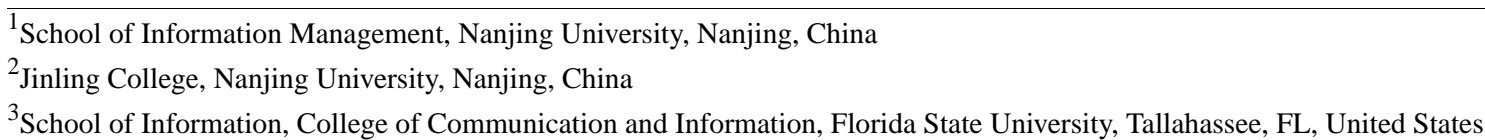

Corresponding Author:

Ying Cheng, PhD

School of Information Management

Nanjing University

163 Xianlin Road

Nanjing, 210023

China

Phone: 8613851838364

Email: chengy@nju.edu.cn

\section{Abstract}

Background: Although previous studies have reported the cancer information-seeking behaviors among patients in high-income countries, the cancer information-seeking practices of patients living in low- and middle-income areas are less known.

Objective: This study investigated the beliefs and information-seeking patterns of cancer patients in southwest China.

Methods: A questionnaire was designed, and data were collected in two hospitals $(\mathrm{N}=285)$ in southwest China. Statistical analyses included bivariate analyses and regressions.

Results: Patients' attitudes towards cancer fatalism were significantly influenced by marital status $(P<.001)$, education $(P<.001)$, and household income $(P<.001)$. Moreover, endorsing fatalistic belief was positively associated with age $(\mathrm{r}=0.35, P<.001)$. The regression model showed that younger patients (odds ratio [OR] 0.96, 95\% CI 0.93-0.99) and those with higher education (OR $1.75,95 \%$ CI 1.09-2.81) were more likely to seek information. Additionally, patients who were less confident in getting information were more likely to find information (OR 1.70, 95\% CI 1.15-2.52), while fatalism belief was not significant in the regression (OR 0.65, 95\% CI 0.22-1.95).

Conclusions: This study explored the information-seeking patterns of cancer patients in southwest China. It was found that many Chinese people endorsed cancer fatalism. These pessimistic beliefs about the potential to prevent and to cure cancer correlate with rather than cause cancer-related information seeking. However, self-efficacy about the confidence in finding needed cancer information was a significant predictor of information-seeking.

(JMIR Cancer 2020;6(2):e16138) doi: $\underline{10.2196 / 16138}$

\section{KEYWORDS}

cancer information seeking; cancer belief; fatalism; southwest China

\section{Introduction}

\section{Background}

According to the estimates of World Health Organization, cancer is the second leading cause of global death and is now responsible for 1 in 6 deaths [1]. Information about cancer can benefit patients in decision making, coping with treatment, their psychological well-being, and their quality of life [2,3]. Studying the patterns of patients' cancer-related information-seeking is thus crucial to high-quality provider-patient communication, improved cancer care, and improved outcomes.

Although previous studies have reported information seeking among cancer patients in high-income countries such as the United States and some European countries [4,5], little is known about cancer-related information patterns among patients living in low- and middle-income regions, where approximately $70 \%$ of deaths from cancer occur [1]. In China, while increasing attention has been paid to cancer control and cancer information dissemination [6], most of the work has been conducted in 
eastern metropolitan regions such as Beijing [7], Hefei [7], and Nanjing [8], leaving cancer patients in the southwest underrepresented. Southwest China consists of 5 provinces and is $14.40 \%$ of China's total population [9]. By 2017, the GDP (gross domestic product) of this region only accounted for $10 \%$ of the total GDP [9]; yet, according to national cancer statistics, southwest China had the highest cancer incidence rates, highest cancer mortality rates, and the lowest cancer survival rates in the country [10]. Possible reasons for these include a higher smoking prevalence, limited medical resources, and inadequate cancer screening and treatment [10]. Thus, the education and cancer care of patients in southwest China are concerning.

People's attitudes and beliefs can influence their intentions and behaviors [11]. For example, beliefs about cancer were associated with help-seeking behavior [12]. Furthermore, it was found that Asian individuals were more likely to have fatalistic cancer beliefs [13], for example, that the outcome of cancer is predetermined and arranged by fortune and predestination, and that nothing can be done to control or change the outcome of cancer [14]. One study [15] explored the role of self-efficacy and found that higher self-efficacy beliefs were positively correlated with cancer-screening intentions. However, most of these studies focused on the general public, while the beliefs among cancer patients, especially those from underrepresented regions, are less known.

\section{Prior Work}

\section{Cancer Patients' Information Seeking}

A seminal work [16] identified the individual differences in cancer information exchange as monitoring and blunting. Monitoring indicated the active seeking of information related to cancer, while blunting indicated the avoidance of threatening information. Later studies [17-19] further categorized active information behavior as information seeking and information scanning, arguing that scanning was a less active behavior that gathered and came across relevant information incidentally. Information scanning often occurred through mass media [20]. Moreover, Lambert, et al [21] delineated patients' cancer-related information-seeking preferences through an in-depth qualitative study, including three types of active search (intense, complementary, and fortuitous) and two types of information avoidance (minimal and guarded). These five patterns differed in essential characteristics such as the type and amount of information needs, as well as sources. A recent study [2] showed that most people were active seekers, although the prevalence of information avoidance was higher than expected.

Based on theoretical frameworks such as the Health Belief Model [22] and the Comprehensive Model of Information Seeking [23], previous studies [19,24-26] have found differences between information seekers and nonseekers among cancer patients. These studies investigated demographic characteristics (eg, gender, age, income, education) first, and then explored the effects of social determinants (such as having a regular health care provider, salience, cancer type, cancer stage, and treatment type); however, among the different studies, the effects of cancer type and cancer stage were often found to be inconsistent.
Comprehensive reviews [27,28] of information need and sources of information among cancer patients have been conducted; the majority of previous studies focused on patients in diagnosis and treatment stages. Therefore, the most prevalent information need was treatment-related, such as a need for information on the side effects of treatments and treatment options [4,28]. Furthermore, the most frequently used information source was health professionals, followed by cancer survivors and the internet $[4,24,29]$. Additionally, the differences for information need and sources used between patients of different age, gender, ethnicity or cancer type have also been explored [19,30,31]. For example, a meta-analysis [30] showed that younger cancer patients need more information.

\section{Cancer Beliefs}

An individual's beliefs about cancer can affect the way that person copes with cancer-related issues. People holding fatalistic or negative beliefs were found to have delayed diagnoses, avoid screening, and have worse survival outcomes [32]. Cancer fatalism has been operationalized by pessimism, helplessness, and confusion about how to prevent cancer. For example, based on the Health Information National Trends Survey (HINTS) [33], Kobayashi and Smith [34] found that a common perception in the US population was that everything caused cancer, one-third did not believe cancer was preventable, and more than half automatically associated cancer with death. Moreover, some research [13,32,35,36] has suggested ethnic and cultural differences in fatalism about cancer prevention, arguing that people from Eastern cultures tend to hold a more fatalistic attitude; they were found to be less likely to engage in cancer-related information-seeking, cancer screening, and cancer-preventive behaviors such as exercising and having a healthy diet. Cancer-related information seeking was also found to have an impact on the beliefs. For example, Lee et al [37] found that among those with a lower education level and less health knowledge, health-related internet use reduced cancer fatalism.

Previous studies [38] related to cancer beliefs focused mainly on the general public. However, little is known about the beliefs of cancer patients or the relationship between patients' beliefs and their health information seeking. A Turkish study [39] found that the majority of cancer patients and half of their relatives considered cancer as curable and preventable, and their beliefs influenced their wish for information and their efforts to obtain it. To improve the quality of the cancer care continuum, more research about cancer beliefs among patients during treatment and survival is necessary.

\section{Goal of This Study}

This study aimed to examine the cancer belief and self-efficacy of cancer patients in southwest China, and the factors influencing their cancer information seeking. A survey was conducted in two hospitals in Chengdu and Meishan. Statistical analyses included bivariate analyses and regression. Findings from this study can help develop effective cancer communication interventions and education strategies to achieve high-quality cancer care. 


\section{Methods}

\section{Overview}

A survey was conducted to explore the cancer beliefs and information seeking of cancer patients in southwest China. The variables of the survey were measured with a self-report questionnaire (Multimedia Appendix 1) which included demographic questions and questions about cancer belief, self-efficacy, and cancer information seeking. The survey was conducted between January 2019 and April 2019.

\section{Recruitment}

Patients with cancer were recruited by convenience sampling in Meishan Tumor Hospital and the oncology inpatient department of the First Affiliated Hospital of Chengdu Medical College, located in Meishan and Chengdu, respectively, in southwest China. The two hospitals vary in levels: Chengdu Medical College is a level 3 hospital and Meishan Tumor Hospital is a level 2 hospital. Eligibility criteria for participation were patients who were diagnosed with cancer and who were undergoing treatment. We did not select patients by cancer type or gender because of ethical considerations required by the hospital administrations. One researcher together with a doctor distributed the questionnaires during the doctor's rounds. Each patient was invited to read and sign an informed consent form, which explained the aims, demands, guaranteed anonymity of the study, and that refusing to participate would not influence treatment. They were also asked to answer the questions carefully and were told that the researcher was available when they needed help.

With the aim of estimating the pattern of patients seeking cancer information in southwest China, we calculated the sample size by referring to a sample size calculation for qualitative variable in cross-sectional studies [40]. A previous study [7] in China showed that the portion of participants who reported having looked for information related to cancer may not be more than $28 \%$, so the target sample size for the current study was determined to be 310 . Three hundred questionnaires were distributed.

\section{Measures}

\section{Cancer Belief}

Cancer belief was assessed according to the HINTS (Health Information National Trends Survey) [41] through a guide question "How much do you agree or disagree with each of the following statements?" followed by 5 items: (1) "It seems like everything causes cancer," (2) "There's not much you can do to lower your chances of getting cancer," (3) "There are so many different recommendations about preventing cancer, it's hard to know which ones to follow," (4) "In adults, cancer is more common than heart disease," (5) "When I think about cancer, I automatically think about death." Responses for each item were rated using a 5-point Likert scale ranging from "strongly disagree" to "strongly agree." The internal consistency of these 5 items was (Cronbach $\alpha=.75$ ). The measurement consisting of 5 items was more reliable (Cronbach $\alpha>$.70) than the measurement with 3 items which has frequently been used in previous studies [34]; therefore, we used the mean of the 5 items to create a fatalism scale. Items adapted from HINTS5 were translated into Mandarin through back translation and group discussion to guarantee linguistic equivalence between the English and Chinese versions.

\section{Self-Efficacy}

Self-efficacy suggests how confident patients are in getting health advice or information they need. From the HINTS [41], we used one item "I am NOT confident in finding cancer-related information I need." The response was rated using a 5-point Likert scale rating ranging from "strongly disagree" to "strongly agree."

\section{Demographic Variables}

Based on previous studies [34,37,42] related to cancer information seeking, and the different information practices among cancer patients with different demographic characteristics, we controlled for demographic variables. These variables included age, marital status (single, never been married; married; widowed; divorced), education (primary school or lower, junior school, high school, some college, bachelor's degree or higher), and household annual income (less than 10,000 RMB or approximately US $\$ 1425 ; 10,000$ to 50,000 RMB; 50,000 to 100,000 RMB; greater than 100,000 RMB). As required by the hospital administrations, we did not collect information on participants' gender and their cancer types to avoid identification.

\section{Dependent Variable}

Cancer information seeking was measured with 1 item "Have you ever looked for information about cancer from any source?" The response was "yes" or "no." Participants who answered "no" were considered nonseekers.

\section{Statistical Analysis}

To investigate the relationship between patients' beliefs and their cancer-related information-seeking behavior, we conducted bivariate analyses as well as multivariate regression analysis. First, the essential characteristics among different group of participants were described. Subsequently, the beliefs and information-seeking differences in demographic variables except age were examined using an analysis of variance (ANOVA) and cross-tabulation (Pearson chi-square test). The relationships between age and beliefs were tested using Pearson correlation while the differences between information seekers and nonseekers were analyzed using two-tailed independent $t$ tests. Moreover, the self-efficacy and cancer belief differences between information seekers and nonseekers were explored using two-tailed independent $t$ tests. Finally, variables were fitted in a logistic regression to investigate their predictive relationship with cancer information-seeking behavior, before which collinearity diagnostics were conducted (Multimedia Appendix 2). The categorical variable (marital status) was transformed into a dummy variable in the regression. All the analyses were conducted in SPSS (version 23.0; IBM Corp) and the significance level was $P<.05$. 


\section{Results}

\section{Descriptive Statistics}

Demographic characteristics of participants $(\mathrm{N}=285)$ are shown in Table 1. Among the 285 patients who completed the survey, the mean age was 51.79 (range 17-95) years old. Most were married $(206 / 285,72.3 \%)$, while the number of patients in the other three categories of marital status were close; and $25.6 \%$ (73/285) reported a junior school level of education, followed by high school, primary school or lower, college, and bachelor's degree or higher. Moreover, 48.4\% (138/285) reported a household annual income lower than 10,000 RMB, while the distribution of patients in the other three income categories was fairly equivalent.

The majority of patients $(233 / 285,81.8 \%)$ had looked for cancer-related information. Cancer belief (mean 3.23, SD 0.52) showed a moderate or strong fatalistic view. Among the 5 types of fatalism, "inevitable death" was most common (mean 3.66, SD 0.95), followed by "helplessness" (mean 3.18, SD 0.75), and "prevalence" (mean 3.17, SD 0.70). Furthermore, confidence in getting cancer information among 108 patients was moderate $(108 / 285,37.9 \%)$, while $6.0 \%$ (17/285) strongly acknowledged their confidence.

Table 1. Participant demographic data.

\begin{tabular}{|c|c|}
\hline Characteristics $(\mathrm{N}=285)$ & Patients, n (\%) \\
\hline \multicolumn{2}{|l|}{ Age } \\
\hline$\leq 19$ & $6(2.1)$ \\
\hline $20-29$ & $23(8.1)$ \\
\hline $30-39$ & $43(15.1)$ \\
\hline $40-49$ & $72(25.3)$ \\
\hline $50-59$ & $50(17.5)$ \\
\hline $60-69$ & $36(12.6)$ \\
\hline $70-79$ & $31(10.9)$ \\
\hline $80-89$ & $20(7.0)$ \\
\hline$\geq 90$ & $4(1.4)$ \\
\hline \multicolumn{2}{|l|}{ Marital status } \\
\hline Single & $26(9.1)$ \\
\hline Married & $206(72.3)$ \\
\hline Widowed & $30(10.5)$ \\
\hline Divorced & $23(8.1)$ \\
\hline \multicolumn{2}{|l|}{ Education } \\
\hline Primary school or lower & $66(23.2)$ \\
\hline Junior school & $73(25.6)$ \\
\hline High school & $67(23.5)$ \\
\hline Some college & $45(15.8)$ \\
\hline Bachelor's degree or higher & $34(11.9)$ \\
\hline \multicolumn{2}{|l|}{ Household income (in $\mathrm{RMB}^{\mathrm{a}}$ ) } \\
\hline$<10,000$ & $138(48.4)$ \\
\hline $10,000-50,000$ & $58(20.4)$ \\
\hline $50,000-100,000$ & $42(14.7)$ \\
\hline$>100,000$ & $47(16.5)$ \\
\hline
\end{tabular}

\footnotetext{
${ }^{\mathrm{a}}$ At the time of publication, an exchange rate of approximately US $\$ 1=0.19 \mathrm{RMB}$ was applicable.
}

\section{Beliefs and Demographics}

We conducted bivariate analyses to investigate the relationship between patients' demographics and their beliefs. Patients' attitudes towards cancer fatalism were significantly related to marital status $(P<.001)$, education $(P<.001)$, and household income $(P<.001)$ (Table 2). Moreover, endorsing fatalistic belief was moderately associated with age $(\mathrm{r}=0.35, P<.001)$. A linear regression analysis was conducted to further test the relationship between demographics and cancer fatalism (Multimedia Appendix 3). It was found that age $(P=.003)$, education 
$(P<.001)$, and household income $(P=.04)$ still showed significant correlation with cancer fatalism. On the other hand, marital status $(P<.001)$, education $(P<.001)$, and household income $(P<.001)$ were also related to patients' self-efficacy. Furthermore, older patients were more likely to believe in their ability to obtain the information they need $(\mathrm{r}=-0.41, P<.001)$. These results were also tested in a linear regression model, in which age $(P<.001)$ and education $(P<.001)$ remained significant (Multimedia Appendix 3).

Table 2. Results of bivariate analyses.

\begin{tabular}{|c|c|c|c|c|}
\hline \multirow[t]{2}{*}{ Variables } & \multicolumn{2}{|l|}{ Cancer belief } & \multicolumn{2}{|l|}{ Self-efficacy } \\
\hline & Value & $P$ value & Value & $P$ value \\
\hline Marital status, mean (SD) & & $<.001^{\mathrm{a}}$ & & $<.001^{\mathrm{a}}$ \\
\hline Single & $2.77(0.44)$ & & $4.08(0.89)$ & \\
\hline Married & $3.23(0.53)$ & & $2.88(1.05)$ & \\
\hline Widowed & $3.47(0.28)$ & & $2.53(0.97)$ & \\
\hline Divorced & $3.44(0.52)$ & & $2.44(1.31)$ & \\
\hline Education, mean (SD) & & $<.001^{\mathrm{a}}$ & & $<.001^{\mathrm{a}}$ \\
\hline Primary school or lower & $3.74(0.37)$ & & $2.38(0.82)$ & \\
\hline Junior school & $3.40(0.34)$ & & $2.47(0.97)$ & \\
\hline High school & $3.11(0.39)$ & & $2.96(1.12)$ & \\
\hline Some college & $2.88(0.35)$ & & $3.44(1.10)$ & \\
\hline Bachelor's degree or higher & $2.69(0.40)$ & & $4.15(0.82)$ & \\
\hline Household annual income, mean (SD) & & $<.001^{\mathrm{a}}$ & & $<.001^{\mathrm{a}}$ \\
\hline$<10,000 \mathrm{RMB}$ & $3.27(0.51)$ & & $2.88(0.99)$ & \\
\hline $10,000-50,000 \mathrm{RMB}$ & $3.28(0.51)$ & & $2.59(1.09)$ & \\
\hline $50,000-100,000 \mathrm{RMB}$ & $3.39(0.44)$ & & $2.67(1.12)$ & \\
\hline$>100,000 \mathrm{RMB}$ & $2.92(0.53)$ & & $3.64(1.19)$ & \\
\hline Age, $r$ & 0.35 & $<.001^{\mathrm{b}}$ & -0.41 & $<.001^{\mathrm{b}}$ \\
\hline
\end{tabular}

${ }^{\mathrm{a}}$ ANOVA.

${ }^{\mathrm{b}}$ Pearson correlation.

\section{Cancer Information Seeking and Demographics}

Our results suggested significant differences between cancer information seekers and nonseekers in marital status $(P<.001)$ and education $(P<.001)$, while household annual income showed no influence $(P=.45)$ (Table 3$)$, and the mean age of nonseekers was significantly higher than that of seekers $(P<.001)$. However, the effect of income became insignificant in the logistic regression (odds ratio [OR] 1.01, 95\% CI 0.68-1.48) (Table 4). In the regression model, education level and age were demographic predictors of cancer information-seeking behavior, with younger patients (OR 0.96, 95\% CI 0.93-0.99) and those with higher education (OR 1.75, 95\% CI 1.09-2.81) being more likely to look for information. 
Table 3. Comparison between patients who seek information and those who do not.

\begin{tabular}{|c|c|c|c|}
\hline \multirow[t]{2}{*}{ Variables } & \multicolumn{3}{|c|}{ Cancer information seeking } \\
\hline & Seekers $(n=233)$ & Nonseekers $(\mathrm{n}=52)$ & $P$ value \\
\hline Marital status, n (\%) & & & $<.001^{\mathrm{a}}$ \\
\hline Single & $26(11.2)$ & $0(0)$ & \\
\hline Married & $173(74.2)$ & $33(63.5)$ & \\
\hline Widowed & $15(6.4)$ & $15(28.8)$ & \\
\hline Divorced & $19(8.1)$ & $4(7.8)$ & \\
\hline Education, $\mathbf{n}(\%)$ & & & $<.001^{\mathrm{a}}$ \\
\hline Primary school or lower & $39(16.7)$ & $27(51.9)$ & \\
\hline Junior school & $58(24.9)$ & $15(28.8)$ & \\
\hline High school & $61(26.2)$ & $6(11.5)$ & \\
\hline Some college & $42(18.0)$ & $3(5.8)$ & \\
\hline Bachelor's degree or higher & $33(14.2)$ & $1(1.9)$ & \\
\hline Household annual income, n (\%) & & & $.45^{\mathrm{a}}$ \\
\hline$<10,000 \mathrm{RMB}$ & $109(46.8)$ & $29(55.8)$ & \\
\hline $10,000-50,000 \mathrm{RMB}$ & $47(20.2)$ & $11(21.2)$ & \\
\hline $50,000-100,000 \mathrm{RMB}$ & $35(15.0)$ & $7(13.5)$ & \\
\hline$>100,000 \mathrm{RMB}$ & $42(18.0)$ & $5(9.6)$ & \\
\hline Age, mean & $48.70(15.97)$ & $65.67(16.91)$ & $<.001^{\mathrm{b}}$ \\
\hline Cancer belief, mean (SD) & $3.16(0.52)$ & $3.56(0.37)$ & $<.001^{\mathrm{b}}$ \\
\hline Self-efficacy, mean (SD) & $3.09(1.04)$ & $2.14(1.14)$ & $<.001^{\mathrm{b}}$ \\
\hline
\end{tabular}

${ }^{\mathrm{a}}$ Pearson chi-square test.

${ }^{\mathrm{b}} t$ test.

Table 4. Results of logistic regression.

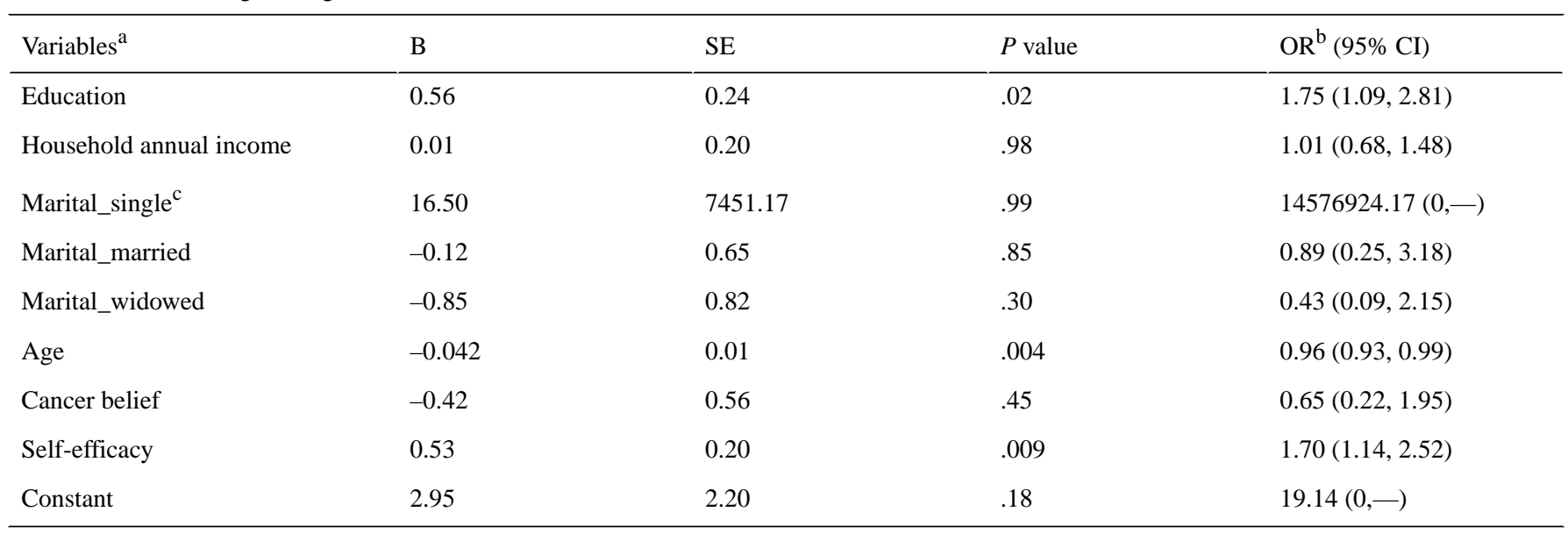

${ }^{\mathrm{a}}$ Model summaries $\left(\right.$ Cox and Snell $\mathrm{R}^{2}=0.24$; Nagelkerke $\left.\mathrm{R}^{2}=0.38\right)$.

${ }^{\mathrm{b}} \mathrm{OR}$ : odds ratio.

${ }^{\mathrm{c}}$ This implausibly large odds ratio value was possibly due to the uneven distribution among marital groups, and it should be interpreted with caution.

\section{Cancer Information Seeking and Beliefs}

Patients' attitudes towards cancer fatalism $(P<.001)$ and their self-efficacy $(P<.001)$ were significantly associated with whether to seek information or not (Table 3). Cancer fatalism was higher http://cancer.jmir.org/2020/2/e16138/ in the nonseeking group (nonseeking: mean 3.56; seeking: mean 3.16) and this group also showed a higher level of confidence (nonseeking: mean 2.14; seeking: mean 3.09; it is notable that in the scale of self-efficacy, a higher value means a greater possibility of agreement that the participant is not confident). 
However, when tested in the regression model, cancer belief was not found to be a predictor for information seeking (OR $0.65,95 \%$ CI $0.22-1.95$ ) (Table 3 ), suggesting a complicated relationship between these two variables. Additionally, patients who were less confident in getting information were more likely to find information (OR 1.70, 95\% CI 1.15-2.52).

\section{Discussion}

\section{Principal Results}

To the best of our knowledge, this is the first study to investigate the beliefs and information-seeking behavior of cancer patients in southwest China. It was found that many endorsed cancer fatalism. These pessimistic beliefs about the potential to prevent and to cure cancer were correlated with rather than the cause of cancer-related information seeking. However, self-efficacy was a significant predictor of seeking out information $(P<.001)$. Besides, it was found that patients who were younger $(P<.001)$ and with higher level of education $(P<.001)$ were more likely to find information. The response rate was quite high $(285 / 300$, $95 \%$ ), which might because (1) we surveyed hospitalized patients, and (2) they were familiar with their doctors and were thus willing to answer the questions.

\section{The Influencing Factors of Cancer Information Seeking}

Cancer fatalism is a multidimensional concept often operationalized by beliefs of pessimism, fear, helplessness, confusion, and inevitable death [39]. A previous study [13] found that people from Asian cultures tend to hold a more fatalistic view on negative issues such as having cancer, considering them to be unpreventable and out of one's control. Moreover, the same results have been reported among Asian communities in Western countries [35]; in these communities, individuals were more likely to associate cancer with bad luck, punishment for sins committed in the current or previous life, or the will of a supreme being [43], embedded in religious traditions such as Taoism. The higher fatalism among cancer patients in Chinese society was supported in our study (mean 3.23 , SD 0.52). In addition, a study [7] found that the average fatalistic attitude of Chinese public was also slightly above the scale midpoint (mean 3.30, SD 0.80, range 1-5). The similar results suggest that cancer care intervention in our study's region failed to dispel fatalistic views. Additionally, in this study, we found that older patients were more likely to endorse fatalistic beliefs, while those who were better educated held more positive perceptions about cancer, as supported by a Turkish research [39].

Although a previous study [36] found that people who held a negative cancer belief were more likely to be information avoiders; our results did not support this: in the logistics model, there was no predictive relationship between cancer belief and information seeking. One possible explanation is that they might influence each other - on one hand, in the literature, it has been reported that greater information seeking led to decreased fatalism [44], internet-use reduced fatalism [37], and negative health information-seeking experiences might contribute to cancer fatalism [45]; on the other hand, some studies [7,46] found that fatalistic attitudes about cancer were an important barrier to information seeking. A significant correlation was found in our bivariate analysis $(P<.001)$, but cancer fatalism was not a predictor in the regression model $(P=.45$; OR 0.65 , $95 \%$ CI $0.22-1.95)$. The bidirectional effects of cancer fatalism and information seeking could be further investigated through a qualitative approach.

In this study, we investigated how confident patients were of their ability to find cancer-related information. Interestingly, we found that older patients were more confident, while those who had higher incomes and were more educated reported to be less confident. One theoretical explanation could be optimistic beliefs about cancer risk among Asians [13]. This belief system allows them to underestimate their chance of suffering from serious outcomes [47]. However, patients with higher socioeconomic status were more likely to be exposed to a more westernized lifestyle and worldview, making them more self-enhancing and self-critical. Thus, older people were found to be more confident than those with higher income and education. On the other hand, as shown in the regression model, patients who were less confident about their information-seeking ability were more likely to look for information. This finding is inconsistent with studies $[48,49]$ that have found that efficacy encourages health information-seeking. The reasons may be two-fold: first, we did not measure self-efficacy through a standard scale as the previous studies did; second, patients reporting less confidence tended to be younger, more educated, and were more likely to seek information. Therefore, the effect of self-efficacy on information seeking deserves future research. Additionally, younger and more educated patients might expect more from the health care system and keep on seeking, since a study found that intense seekers were more likely to be dissatisfied with the cancer information provided [2].

A large percentage of our respondents reported a low household income and low education level, which suggests social and economic gaps between the eastern and western China. For example, in two eastern cities (Beijing and Hefei, Anhui province), the majority of households earn 60,001 RMB or more annually [7], while most participants in this study had an annual household income of 10,000 RMB or lower.

Our analyses also revealed distinct patterns in the information-seeking behavior among different groups of cancer patients. We found that older patients and those who were less educated were less likely to seek information, which is consistent with a previous study [26] conducted in Mexico. This study focused on health-related information-seeking behaviors and preferences among Mexican patients with cancer, showing that older age was the characteristic most strongly associated with not seeking information [26]. Moreover, similar results have been found in some high-income countries; Smith-McLallen et al [50] found that level of education and age significantly contributed to the prediction of patients' seeking intentions in Pennsylvania, while they also indicated that older individuals and those who had lower levels of education were less likely to seek out information from sources other than their doctors. It was also found in a study [2] in Montreal that individuals who avoided information tended to be less educated, while active seekers reported higher education. However, a study [7] in Beijing and Hefei found that age and employment were not related to seeking. Moreover, although education 
showed some significant relationships with seeking in the predictive models considering predisposing and enabling variables, the relationship became nonsignificant when including need variables [7].

\section{Practical Suggestions}

Given the importance of information seeking to cancer care and outcomes, health practitioners should encourage patients' information seeking behavior and increase their satisfaction. First, education was found to be an important predictor of information seeking, highlighting the importance of narrowing educational gaps together with economic gaps between southwestern and eastern regions in China. This could generally increase patients' health literacy since education and health literacy often overlap [34]. Moreover, many Chinese individuals hold the fatalistic beliefs about cancer. Practitioners should understand the roles of these beliefs better before attempting interventions to improve the access and availability of optimal treatments. For example, the relationship between cancer beliefs and traditional Chinese medicine should be considered. Furthermore, cancer patients undergoing treatment in the hospital are seen by the same fellow or oncologist, which facilitates a steady and trustable relationship. This relationship can form a close tie, which is a fundamental value in the Chinese collectivism culture, to benefit information provision. For example, during hospital stays, oncologists can provide tailored messages, tell the patients how to find information, and guide them in appraising information sources. In addition, oncologists may also continue to help patients in their information seeking by using email and social networking sites based on the close relationships.

\section{Limitations}

There are some limitations in our study. For example, this cross-sectional survey was not equipped to explore the causality between variables. Furthermore, this study only investigated several possible factors influencing beliefs and information seeking. Further research is needed to follow a theoretical framework and explore more factors. This survey was conducted in two hospitals in southwest China, and thus may not be generalizable outside of a similar context. Finally, since the participants of all types of cancer were randomly sampled, the results could be biased by the participants with the majority type of cancer and should be interpreted with caution.

\section{Conclusions}

This study explored the information-seeking patterns of cancer patients in southwest China. It was found that many Chinese people endorsed cancer fatalism. These pessimistic beliefs about the potential to prevent and to cure cancer are correlated with cancer-related information seeking, while self-efficacy confidence in finding needed cancer information was a significant predictor of seeking out information. Moreover, this study demonstrated that information seekers and nonseekers differ demographically. These findings reflect a sense of urgency for cancer information dissemination in low- to middle-income regions such as southwest China.

\section{Acknowledgments}

This study was financially supported by a project of Humanities and Social Sciences in Nanjing University (No. 2018047).

\section{Conflicts of Interest}

None declared.

\section{Multimedia Appendix 1}

Questionnaire_English version.

[DOCX File, 24 KB-Multimedia Appendix 1]

\section{Multimedia Appendix 2}

The results of collinearity diagnosis.

[DOCX File , 22 KB-Multimedia Appendix 2]

\section{Multimedia Appendix 3}

Results of two linear regressions.

[DOCX File, 22 KB-Multimedia Appendix 3]

\section{References}

1. World Health Organization. 2018. Cancer. URL: https://www.who.int/news-room/fact-sheets/detail/cancer [accessed 2020-07-30]

2. Loiselle CG. Cancer information-seeking preferences linked to distinct patient experiences and differential satisfaction with cancer care. Patient Educ Couns 2019 Jun;102(6):1187-1193. [doi: 10.1016/j.pec.2019.01.009] [Medline: 30685191]

3. Eisfeld H, Bauer F, Dubois C, Schmidt T, Kastrati K, Hochhaus A, et al. Importance of and Satisfaction with Information about Their Disease in Cancer Patients. J Cancer Educ 2019 Jan 25. [doi: 10.1007/s13187-019-1480-7] [Medline: 30684231] 
4. Rutten LJF, Agunwamba AA, Wilson P, Chawla N, Vieux S, Blanch-Hartigan D, et al. Cancer-Related Information Seeking Among Cancer Survivors: Trends Over a Decade (2003-2013). J Cancer Educ 2016 Jun;31(2):348-357. [doi: 10.1007/s13187-015-0802-7] [Medline: 25712202]

5. Vogel BA, Bengel J, Helmes AW. Information and decision making: patients' needs and experiences in the course of breast cancer treatment. Patient Educ Couns 2008 Apr;71(1):79-85. [doi: 10.1016/j.pec.2007.11.023] [Medline: 18191933]

6. Wen C. China's plans to curb cervical cancer. Lancet Oncol 2005 Mar;6(3):139-141. [doi: 10.1016/S1470-2045(05)01761-4] [Medline: 15737830$]$

7. Zhao X, Mao Q, Kreps GL, Yu G, Li Y, Chou SW, et al. Cancer information seekers in china: a preliminary profile. J Health Commun 2015;20(5):616-626. [doi: 10.1080/10810730.2015.1012244] [Medline: 25845031]

8. Zhang Y, Wen N, Chao N. Effects of mobile information-seeking on the intention to obtain reproductive cancer screening among chinese women: testing an integrative model. Chinese Journal of Communication 2018 Dec 22;12(1):102-121. [doi: $10.1080 / 17544750.2018 .1528291]$

9. National Health Commission of PRC. China Health Statistic Annual. Beijing: Peking Union Medical College Press; 2018.

10. Chen W, Zheng R, Baade PD, Zhang S, Zeng H, Bray F, et al. Cancer statistics in China, 2015. CA Cancer J Clin 2016;66(2):115-132 [FREE Full text] [doi: 10.3322/caac.21338] [Medline: 26808342]

11. Ajzen I. The theory of planned behavior. Organizational Behavior and Human Decision Processes 1991 Dec;50(2):179-211. [doi: 10.1016/0749-5978(91)90020-T]

12. Pedersen AF, Forbes L, Brain K, Hvidberg L, Wulff CN, Lagerlund M, et al. Negative cancer beliefs, recognition of cancer symptoms and anticipated time to help-seeking: an international cancer benchmarking partnership (ICBP) study. BMC Cancer 2018 Apr 02;18(1):363 [FREE Full text] [doi: 10.1186/s12885-018-4287-8] [Medline: 29609534]

13. Kim HK, Lwin MO. Cultural Effects on Cancer Prevention Behaviors: Fatalistic Cancer Beliefs and Risk Optimism Among Asians in Singapore. Health Commun 2017 Oct;32(10):1201-1209. [doi: 10.1080/10410236.2016.1214224] [Medline: 27613075]

14. Cheng H, Sit JWH, Twinn SF, Cheng KKF, Thorne S. Coping with breast cancer survivorship in Chinese women: the role of fatalism or fatalistic voluntarism. Cancer Nurs 2013;36(3):236-244. [doi: 10.1097/NCC.0b013e31826542b2] [Medline: $\underline{22964870]}$

15. Lipkus IM, Johnson C, Amarasekara S, Pan W, Updegraff JA. Predicting Colorectal Cancer Screening among Adults Who Have Never Been Screened: Testing the Interaction between Message Framing and Tailored Risk Feedback. J Health Commun 2019;24(3):262-270. [doi: 10.1080/10810730.2019.1597950] [Medline: 30958101]

16. Miller SM. Monitoring versus blunting styles of coping with cancer influence the information patients want and need about their disease. Implications for cancer screening and management. Cancer 1995 Jul 15;76(2):167-177. [Medline: 8625088]

17. Shim M, Kelly B, Hornik R. Cancer information scanning and seeking behavior is associated with knowledge, lifestyle choices, and screening. J Health Commun 2006;11 Suppl 1:157-172. [doi: 10.1080/10810730600637475] [Medline: 16641081]

18. Niederdeppe J, Hornik RC, Kelly BJ, Frosch DL, Romantan A, Stevens RS, et al. Examining the dimensions of cancer-related information seeking and scanning behavior. Health Commun 2007;22(2):153-167. [doi: 10.1080/10410230701454189] [Medline: 17668995]

19. Nagler RH, Gray SW, Romantan A, Kelly BJ, DeMichele A, Armstrong K, et al. Differences in information seeking among breast, prostate, and colorectal cancer patients: results from a population-based survey. Patient Educ Couns 2010 Dec;81 Suppl:S54-S62 [FREE Full text] [doi: 10.1016/j.pec.2010.09.010] [Medline: 20934297]

20. Ruppel EK. Scanning Health Information Sources: Applying and Extending the Comprehensive Model of Information Seeking. J Health Commun 2016;21(2):208-216. [doi: 10.1080/10810730.2015.1058438] [Medline: 26716985]

21. Lambert SD, Loiselle CG, Macdonald ME. An in-depth exploration of information-seeking behavior among individuals with cancer: part 1: understanding differential patterns of active information seeking. Cancer Nurs 2009;32(1):11-23; quiz 24. [doi: 10.1097/01.NCC.0000343372.24517.bd] [Medline: 19104197]

22. Rosenstock IM. Historical Origins of the Health Belief Model. Health Educ Behav 1974 Dec 01;2(4):328-335. [doi: $\underline{10.1177 / 109019817400200403]}$

23. Johnson J, Meischke H. A Comprehensive Model of Cancer-Related Information Seeking Applied to Magazines. Human Comm Res 1993 Mar;19(3):343-367. [doi: 10.1111/j.1468-2958.1993.tb00305.x]

24. Mayer DK, Terrin NC, Kreps GL, Menon U, McCance K, Parsons SK, et al. Cancer survivors information seeking behaviors: a comparison of survivors who do and do not seek information about cancer. Patient Educ Couns 2007 Mar;65(3):342-350. [doi: 10.1016/j.pec.2006.08.015] [Medline: 17029864]

25. Hartoonian N, Ormseth SR, Hanson ER, Bantum EO, Owen JE. Information-seeking in cancer survivors: application of the Comprehensive Model of Information Seeking to HINTS 2007 data. J Health Commun 2014;19(11):1308-1325. [doi: 10.1080/10810730.2013.872730] [Medline: 24742287]

26. Soto-Perez-de-Celis E, Perez-Montessoro V, Rojo-Castillo P, Chavarri-Guerra Y. Health-Related Information-Seeking Behaviors and Preferences Among Mexican Patients with Cancer. J Cancer Educ 2018 Jun;33(3):505-509. [doi: 10.1007/s13187-018-1334-8] [Medline: 29442291] 
27. Rutten LJF, Arora NK, Bakos AD, Aziz N, Rowland J. Information needs and sources of information among cancer patients: a systematic review of research (1980-2003). Patient Educ Couns 2005 Jun;57(3):250-261. [doi: 10.1016/j.pec.2004.06.006] [Medline: 15893206]

28. Fletcher C, Flight I, Chapman J, Fennell K, Wilson C. The information needs of adult cancer survivors across the cancer continuum: A scoping review. Patient Educ Couns 2017 Mar;100(3):383-410. [doi: 10.1016/j.pec.2016.10.008] [Medline: $\underline{27765377]}$

29. Miller KA, Ramirez CN, Wojcik KY, Ritt-Olson A, Baezconde-Garbanati L, Thomas SM, et al. Prevalence and correlates of health information-seeking among Hispanic and non-Hispanic childhood cancer survivors. Support Care Cancer 2018 Apr;26(4):1305-1313 [FREE Full text] [doi: 10.1007/s00520-017-3956-5] [Medline: 29124416]

30. Ankem K. Factors influencing information needs among cancer patients: A meta-analysis. Library \& Information Science Research 2006 Mar;28(1):7-23. [doi: 10.1016/j.lisr.2005.11.003]

31. Squiers L, Finney RLJ, Treiman K, Bright MA, Hesse B. Cancer patients' information needs across the cancer care continuum: evidence from the cancer information service. J Health Commun 2005; 10 Suppl 1:15-34. [doi: 10.1080/10810730500263620] [Medline: 16377598 ]

32. Min HS, Park J, Kim YA, Yang HK, Park K. Income Difference in Attitudes towards Cancer in General Population: Findings from a National Survey. J Korean Med Sci 2018 Aug 13;33(33):e215 [FREE Full text] [doi: 10.3346/jkms.2018.33.e215] [Medline: $\underline{30093846}$ ]

33. National Cancer Institute. 2013. Health information national trends survey 4 cycle 3. URL: http://hints.cancer.gov [accessed 2020-07-30]

34. Kobayashi LC, Smith SG. Cancer Fatalism, Literacy, and Cancer Information Seeking in the American Public. Health Educ Behav 2016 Aug;43(4):461-470 [FREE Full text] [doi: 10.1177/1090198115604616] [Medline: 26377524]

35. Licqurish S, Phillipson L, Chiang P, Walker J, Walter F, Emery J. Cancer beliefs in ethnic minority populations: a review and meta-synthesis of qualitative studies. Eur J Cancer Care (Engl) 2017 Jan;26(1). [doi: 10.1111/ecc.12556] [Medline: 27515153]

36. Nguyen GT, Bellamy SL. Cancer information seeking preferences and experiences: disparities between Asian Americans and Whites in the Health Information National Trends Survey (HINTS). J Health Commun 2006;11 Suppl 1:173-180. [doi: 10.1080/10810730600639620] [Medline: 16641082]

37. Lee C, Niederdeppe J, Freres D. Socioeconomic Disparities in Fatalistic Beliefs About Cancer Prevention and the Internet. J Commun 2012 Dec;62(6):972-990 [FREE Full text] [doi: 10.1111/j.1460-2466.2012.01683.x] [Medline: 25530627]

38. Sinky TH, Faith J, Lindly O, Thorburn S. Cancer Fatalism and Preferred Sources of Cancer Information: an Assessment Using 2012 HINTS Data. J Cancer Educ 2018 Feb;33(1):231-237. [doi: 10.1007/s13187-016-1115-1] [Medline: 27650861]

39. Kav S, Tokdemir G, Tasdemir R, Yalili A, Dinc D. Patients with cancer and their relatives beliefs, information needs and information-seeking behavior about cancer and treatment. Asian Pac J Cancer Prev 2012;13(12):6027-6032 [FREE Full text] [doi: 10.7314/apjcp.2012.13.12.6027] [Medline: 23464398]

40. Charan J, Biswas T. How to calculate sample size for different study designs in medical research? Indian J Psychol Med 2013 Apr;35(2):121-126 [FREE Full text] [doi: 10.4103/0253-7176.116232] [Medline: 24049221]

41. National Cancer Institute. 2017. Health information national trends survey 5 cycle 1. URL: http://hints.cancer.gov [accessed 2020-07-30]

42. Halbach SM, Ernstmann N, Kowalski C, Pfaff H, Pförtner T, Wesselmann S, et al. Unmet information needs and limited health literacy in newly diagnosed breast cancer patients over the course of cancer treatment. Patient Educ Couns 2016 Sep;99(9):1511-1518. [doi: 10.1016/j.pec.2016.06.028] [Medline: 27378079]

43. Ong KJ, Back MF, Lu JJ, Shakespeare TS, Wynne CJ. Cultural attitudes to cancer management in traditional South-East Asian patients. Australas Radiol 2002 Dec;46(4):370-374. [doi: 10.1046/j.1440-1673.2002.t01-1-01085.x] [Medline: $\underline{12452906]}$

44. Valera P, Lian Z, Brotzman L, Reid A. Fatalistic Cancer Beliefs and Information Seeking in Formerly Incarcerated African-American and Hispanic Men: Implications for Cancer Health Communication and Research. Health Commun 2018 May;33(5):576-584 [FREE Full text] [doi: 10.1080/10410236.2017.1283564] [Medline: 28278604]

45. Emanuel AS, Godinho CA, Steinman C, Updegraff JA. Education differences in cancer fatalism: The role of information-seeking experiences. J Health Psychol 2018 Oct;23(12):1533-1544. [doi: 10.1177/1359105316664129] [Medline: 27553609]

46. Wigfall LT, Friedman DB. Cancer Information Seeking and Cancer-Related Health Outcomes: A Scoping Review of the Health Information National Trends Survey Literature. J Health Commun 2016 Sep;21(9):989-1005 [FREE Full text] [doi: 10.1080/10810730.2016.1184358] [Medline: 27466828]

47. Ji LJ, Zhang Z, Usborne E, Guan Y. Optimism across cultures: In response to the severe acute respiratory syndrome outbreak. Asian Journal of Social Psychology 2004;7(1):25-34. [doi: 10.1111/j.1467-839X.2004.00132.x]

48. Selsky C, Luta G, Noone A, Huerta EE, Mandelblatt JS. Internet access and online cancer information seeking among Latino immigrants from safety net clinics. J Health Commun 2013;18(1):58-70 [FREE Full text] [doi:

10.1080/10810730.2012.688248] [Medline: 23066874] 
49. Lim S, Xue L, Yen CC, Chang L, Chan HC, Tai BC, et al. A study on Singaporean women's acceptance of using mobile phones to seek health information. Int J Med Inform 2011 Dec;80(12):e189-e202. [doi: 10.1016/j.ijmedinf.2011.08.007] [Medline: 21956003]

50. Smith-McLallen A, Fishbein M, Hornik RC. Psychosocial determinants of cancer-related information seeking among cancer patients. J Health Commun 2011 Feb;16(2):212-225 [FRE Full text] [doi: 10.1080/10810730.2010.522227] [Medline: $\underline{21207310}$ ]

\section{Abbreviations}

ANOVA: analysis of variance

GDP: gross domestic product

HINTS: Health Information National Trends Survey

RMB: Renminbi

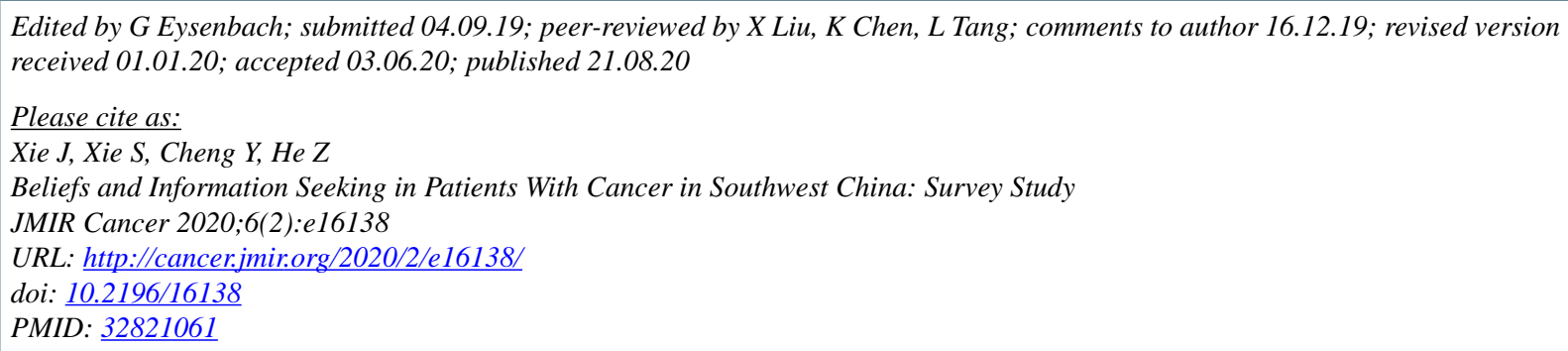

(CJuan Xie, Shi Xie, Ying Cheng, Zhe He. Originally published in JMIR Cancer (http://cancer.jmir.org), 21.08.2020. This is an open-access article distributed under the terms of the Creative Commons Attribution License (https://creativecommons.org/licenses/by/4.0/), which permits unrestricted use, distribution, and reproduction in any medium, provided the original work, first published in JMIR Cancer, is properly cited. The complete bibliographic information, a link to the original publication on http://cancer.jmir.org/, as well as this copyright and license information must be included. 